\title{
Dual functional molecular imaging probe targeting CD20 with PET and optical imaging
}

\author{
PRAMILA PAUDYAL ${ }^{1}$, BISHNUHARI PAUDYAL ${ }^{1}$, YASUHIKO IIDA ${ }^{2}$, NOBORU ORIUCHI ${ }^{1}$, \\ HIROFUMI HANAOKA ${ }^{2}$, HIDEYUKI TOMINAGA ${ }^{1}$, TOMOHIRO ISHIKITA ${ }^{1}$, \\ HIROKI YOSHIOKA ${ }^{1}$, TETSUYA HIGUCHI ${ }^{1}$ and KEIGO ENDO ${ }^{1}$ \\ ${ }^{1}$ Departments of Diagnostic Radiology and Nuclear Medicine, ${ }^{2}$ Bioimaging Information Analysis, \\ Gunma University Graduate School of Medicine, 3-39-22 Showa-machi, Maebashi, Gunma 371-0035, Japan
}

Received February 25, 2009; Accepted April 2, 2009

DOI: 10.3892/or_00000413

\begin{abstract}
The present study aimed to develop a monoclonal antibody (mAb)-based double functional probe for PET and near-infrared fluorescence (NIRF) targeting CD20 and to cross validate the targeting efficacy of this dual functional probe. NuB2, anti CD20 mAb was conjugated with Alexa Fluor 750 and ${ }^{64} \mathrm{Cu}$ through DOTA chelator. PET and NIRF imaging was carried out at $30 \mathrm{~min}$ and $24 \mathrm{~h}$ post-injection with ${ }^{64} \mathrm{Cu}$-DOTA-NuB2-Alexa Fluor 750 in CD20 positive Raji lymphoma-bearing mice. Fluorescence intensity and radio-activity were studied by ex vivo biodistribution study at $30 \mathrm{~min}, 24$ and $48 \mathrm{~h}$ after injection. Raji tumor showed significantly higher uptake of DOTA-NuB2-Alexa Fluor 750 than that of DOTA-Alexa Fluor $750(\mathrm{p}<0.05)$. Significant correlation was obtained between the organ-to-muscle ratios measured by the radioactivity and fluorescence intensity $(\mathrm{p}<2.2 \mathrm{e}-16, \mathrm{r}=0.94)$. Our findings demonstrate the effectiveness and feasibility of preparing an effective mAbbased dual functional imaging agent for PET and NIRF targeting the CD20 expression in lymphoma.
\end{abstract}

\section{Introduction}

Near infrared fluorescence (NIRF) imaging is promising in the field of optical imaging as it offers unique advantage for diagnostic imaging of solid tumors with high sensitivity (1). NIR light with wavelength of 700-900 nm causes minimal autofluorescence and is minimally absorbed by hemoglobin as well as by water and lipids $(2,3)$. On the other hand, positron emission tomography (PET) is an established, highly sensitive

Correspondence to: Dr Pramila Paudyal, Department of Diagnostic Radiology and Nuclear Medicine, Gunma University Graduate School of Medicine, 3-39-22 Showa-machi, Maebashi, Gunma 371-8511, Japan

E-mail:pramila@med.gunma-u.ac.jp

Key words: ${ }^{64} \mathrm{Cu}, \mathrm{PET}$, near infrared fluorescence, CD20, lymphoma, monoclonal antibody and a quantitative tomographic imaging modality (4). However, radiotracers have the limitation of low photon counts thus requiring longer scan times as well as they have definite halflives and the radiation dosage which prevents the longitudinal imaging in patients. Although NIRF and radiotracers possess excellent photophysical properties, none are tumor specific. Radiolabeled or fluorescence labeled antibodies/peptides have been vigorously investigated to overcome these problems. By combining optical imaging for enhanced photon signal count without agent half-life concerns and PET imaging with high sensitivity at deep tissue detection, mAb based dual-labeled imaging agent allows cross validation and direct comparison between nuclear and optical imaging and allows a comprehensive picture of the imaging agent distribution and provides a rapid and efficient imaging method for basic research and clinical diagnosis (4-7).

Few studies on dual-labeled probe for imaging has been reported so far. Protein or peptide was dual-labeled as an imaging agent for PET and NIRF $(8,9)$. Another study reported dual labeled monoclonal antibody (mAb)-based imaging agent for SPECT and NIRF (10). ${ }^{64} \mathrm{Cu}$-labeled peptides have also shown promise for PET and targeted radiotherapy in tumor-bearing animals and for diagnostic imaging of patients $(4,11)$. Although radiolabeled peptides accumulate within few hours in tumor unlike mAb, the absolute tumor uptake is generally considerably less than that with labeled mAb. Radiolabeled mAb display more rapid pharmacokinetics and impressive outcome. Excellent promising results have been obtained with radiolabeled anti-CD20 mAb in the treatment of non-Hodgkin's lymphoma (12).

To the best of our knowledge no study has been reported so far in dual labeled mAb-based imaging agent for PET and NIRF. The aim of the present study is to develop a monomolecular multimodal imaging agent for PET and NIRF targeting CD20 positive lymphoma that will have better targeting ability, good pharmacokinetics and better imaging and would be able to cross validate the findings of PET and NIRF modalities.

\section{Materials and methods}

Cell lines. Human lymphoma cell lines Raji was purchased from American Type Culture (ATCC, Rockville, USA) and 
was cultured in RPMI-1640 medium (Sigma, St. Louis, MO, USA) supplemented with $10 \%$ fetal bovine serum and antibiotics (100 $\mathrm{U} \mathrm{ml}^{-1}$ penicillin and $100 \mu \mathrm{g} \mathrm{ml}^{-1}$ streptomycin).

DOTA-NuB2 synthesis. Anti-CD20 murine mAb, NuB2 (kindly provided by Immunobiological Laboratory, Gunma, Japan), was conjugated with 1,4,7,10-tetraazacyclododecane1, 4, 7, 10-tetracetic acid (mDOTA). To a solution of mDOTA $(0.3 \mathrm{mg}, 20$ molar excess) in DMSO $(5 \mu \mathrm{l})$, NuB2 (10 mg/ $\mathrm{ml}$ ) was added to $500 \mu 1$ borate buffered saline $(0.1 \mathrm{M}, \mathrm{pH} 8.5)$ and the mixture was incubated overnight at room temperature. The crude antibody was purified using Bio-Spin ${ }^{\circledR} 6$ Tris column (Bio-Rad, CA, USA) using phosphate buffer as the mobile phase.

DOTA-NuB2 conjugation with Alexa Fluor 750. DOTA-NuB2 conjugation with Alexa Fluor 750 was done with SAIVI ${ }^{\mathrm{TM}}$ Rapid antibody labeling kits (Invitrogen Corp., Carlsbad, CA, USA) according to the manufacturer's protocol. Briefly, $2 \mathrm{mg} / \mathrm{ml}$ of the DOTA-NuB2 was mixed with sodium bicarbonate and conjugated with Alexa Fluor 750. The purification of labeled protein was performed in a spin column filled with size exclusion resin in PBS, $\mathrm{pH}$ 7.2. Spectrophotometer was used to analyze the moles of dye-to-protein from absorbance values 280 and $750 \mathrm{~nm}$ at UV region.

Radiolabeling. ${ }^{64} \mathrm{Cu}$ was synthesized by in-house cyclotron (kindly provided by Japan Atomic Energy Association, Takasaki, Japan). DOTA-NuB2 conjugated with Alexa Fluor 750 was labeled with ${ }^{64} \mathrm{CuCl}_{2}$. To the solution of acetic buffer (3 M, pH 6.0), $50 \mu \mathrm{g}$ of DOTA-NuB2-Alexa Fluor 750 was added and was radiolabeled with ${ }^{64} \mathrm{CuCl}_{2}$ and the mixture was incubated at $40{ }^{\circ} \mathrm{C}$ for 1.5 h. ${ }^{64} \mathrm{Cu}$-DOTA-NuB2-Alexa Fluor 750 was purified by Bio-Spin 6 Tris column using phosphate buffer as the mobile phase. The radiochemical purity was determined by thin-layered chromatography.

Biodistribution study in tumor-bearing mice. Animal experiments were conducted in accordance with our institutional guideline and were approved by the Gunma University Animal Care Committee. Balb/c nude mice, 6 weeks old, female were purchased from CLEA (Tokyo, Japan) and were maintained under specific pathogen-free conditions and were provided with sterile food and water. Nude mice were inoculated subcutaneously with CD20 expressing Raji cells. When the tumors were $\sim 7-8 \mathrm{~mm}$ in diameter, the animals were injected intravenously with ${ }^{64} \mathrm{Cu}$-DOTA-NuB2-Alexa Fluor $750(10 \mathrm{MBq} /$ mouse). Group of 4 mice were used for the experiments. At 24 and $48 \mathrm{~h}$ after injection, mice were sacrificed by decapitation and aliquots of blood were collected. Organs of interest were excised and weighed, and the radioactivity counts was measured with a well counter (ARC-7001, Aloka, Tokyo, Japan). The data are expressed as \% injected dose/gram (ID/g).

Small animal PET scan and image analysis. PET imaging of Raji xenografts was performed by PET scanner (Inveon, Siemens, IL, USA). The scanner has computer-controlled vertical and horizontal bed motion, with an effective axial field of view (FOV) of $7.8 \mathrm{~cm}$ and a transaxial FOV of $10 \mathrm{~cm}$.
The mice were injected with $\sim 5-8 \mathrm{MBq}$ of ${ }^{64} \mathrm{Cu}$-DOTA$\mathrm{NuB} 2-$ Alexa Fluor 750 via the tail vein and then anesthetized with $1 \%$ Nembutal and placed in the prone position near the center of the FOV of the PET scanner where the highest image resolution and sensitivity are obtained. The $10 \mathrm{~min}$ static scans were obtained for every mouse at $30 \mathrm{~min}$ and $24 \mathrm{~h}$. The images were reconstructed by a 2 -dimensional ordered-subsets expectation maximization algorithm. No correction was done for attenuation and scattering.

Fluorescence imaging. Fluorescence imaging was performed with small animal in vivo imaging system (Maestro, CRI, MA, USA) at $30 \mathrm{~min}$ and $24 \mathrm{~h}$ post-injection with ${ }^{64} \mathrm{Cu}$ DOTA-NuB2-Alexa Fluor 750 or DOTA-Alexa Fluor 750. The system consists of an optical head that includes a liquid crystal tunable filter with a bandwidth of $20 \mathrm{~nm}$ and a scanning wavelength range of 500-950 $\mathrm{nm}$ with a custom designed, spectrally optimized lens system that relays the image to a scientific-grade megapixel CCD. The tunable filter was automatically stepped in $10 \mathrm{~nm}$ increments from 650 to $850 \mathrm{~nm}$ while the camera captured images at each wavelength with constant $1 \mathrm{sec}$ exposure (total acquisition time was $25-30 \mathrm{sec}$ ). The resulting 21 TIFF images (spectral cube, containing a spectrum at every pixel) were loaded into vendor software (Nuance 1.4.0) and analyzed. Spectral unmixing using a normal mouse as the autofluorescence signal yielded the pseudo color images of the pure mice autofluorescence and the Alexa Fluor 750. The animals were anesthetized with Nembutal before the acquisition was started.

For ex vivo studies, the excised tissues were subjected to fluorescence imaging. The fluorescence intensity of each tissue was measured and normalized to photons per second with an ROI covering the entire tissue. The total fluorescence flux of each tissue was divided by its weight. The data were calculated as $\% \mathrm{ID} / \mathrm{g}$.

Statistical analysis. All statistical analysis was performed with Stat View 5.0. Spearman's correlation was performed between organ-to-muscle ratios of PET and NIRF. The Kruskal Wallis test was performed to calculate the difference between the variables.

\section{Results}

Synthesis of ${ }^{64} \mathrm{Cu}$-DOTA-NuB2-Alexa Fluor 750. The radiochemical purity of ${ }^{64} \mathrm{Cu}-\mathrm{DOTA}-\mathrm{NuB} 2-\mathrm{Alexa}$ Fluor 750 was $80 \%$. The number of DOTA chelators bound to a single molecule of NuB2 was found to be $\sim 10$. The absorption and fluorescence emission spectra of ${ }^{64} \mathrm{Cu}$-DOTA-NuB2-Alexa Fluor 750 and DOTA-NuB2-Alexa Fluor 750 were similar to Alexa Fluor 750 suggesting that the fluorescence properties of the Alexa Fluor 750 were not altered on conjugation with the antibody. The number of Alexa Fluor 750 conjugated to the DOTA-NuB2 was determined by spectrophotometer and the mole of dye to antibody ratio was $\sim 2$.

In vivo small animal PET and optical imaging. Fig. 1a-f shows PET and NIRF imaging at $30 \mathrm{~min}$ and $24 \mathrm{~h}$ postinjection of ${ }^{64} \mathrm{Cu}$-DOTA-NuB2-Alexa Fluor 750 or DOTAAlexa Fluor 750. Mouse injected with DOTA-Alexa Fluor 

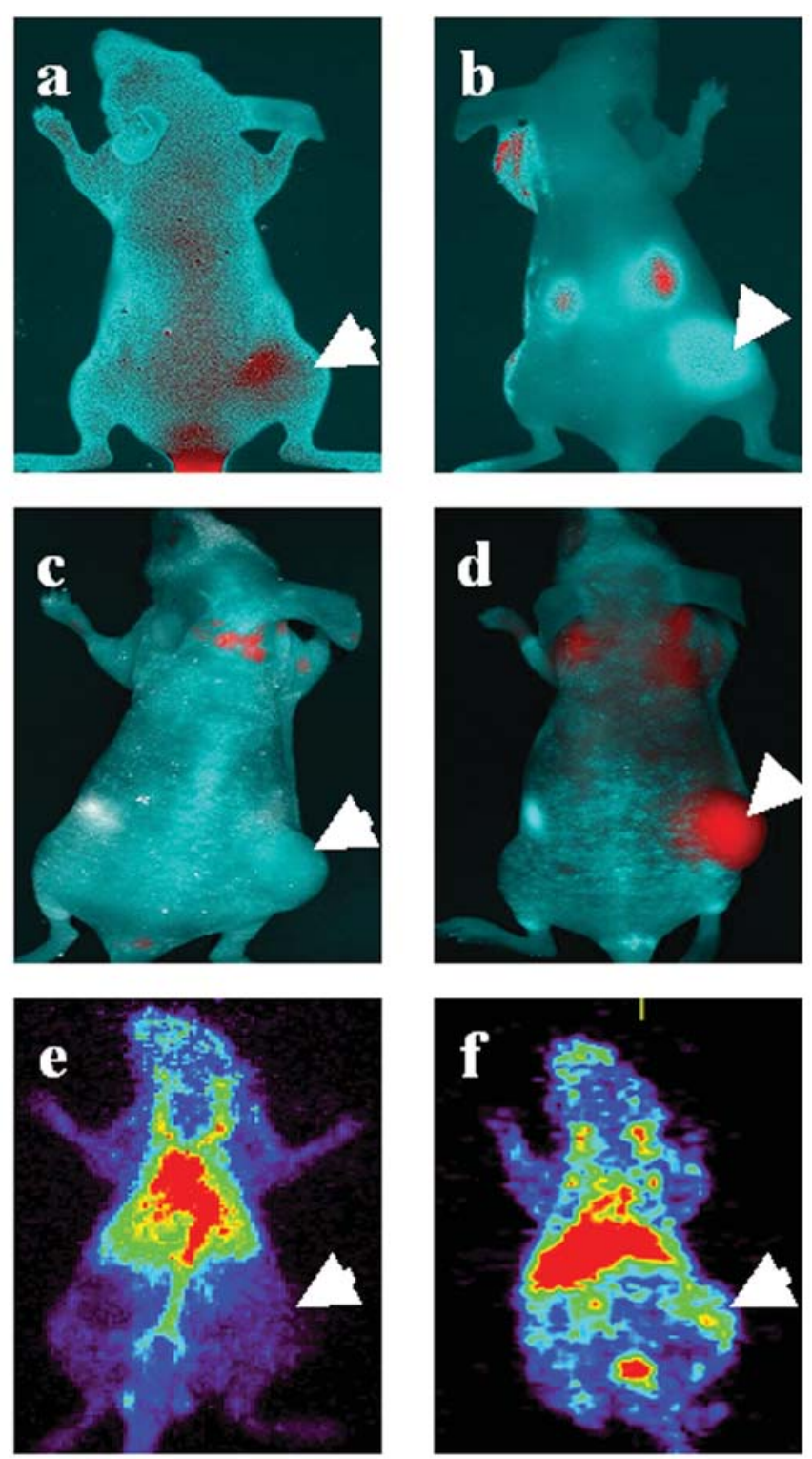

Figure 1. Whole body PET and NIRF images of Raji xenografts. (a) NIRF imaging after 30-min post-injection with DOTA-Alexa Fluor 750 and (b) NIRF imaging after $24 \mathrm{~h}$ (c) NIRF imaging after 30 min post-injection with ${ }^{64} \mathrm{Cu}$-DOTA-NuB2-Alexa Fluor 750 and (d) NIRF imaging after $24 \mathrm{~h}$. Red color indicates Alexa Fluor 750 and cyan color indicates autofluorescene (e) PET image after 30 min post-injection with ${ }^{64} \mathrm{Cu}$-DOTA-NuB2-Alexa Fluor 750 and (f) PET imaging after $24 \mathrm{~h}$. Arrow indicates the tumor.

750 did not show any uptake in the tumor at $30 \mathrm{~min}$ and $24 \mathrm{~h}$ post-injection (Fig. 1a and b). Similarly, no uptake of ${ }^{64} \mathrm{Cu}-$ DOTA-NuB2-Alexa Fluor 750 is seen in the tumor at $30 \mathrm{~min}$ with PET and NIRF (Fig. $1 \mathrm{c}$ and e) whereas significant uptake of ${ }^{64} \mathrm{Cu}$-DOTA-NuB2-Alexa Fluor 750 is seen at the tumor region after $24 \mathrm{~h}$ post-injection as determined by the NIRF and PET imaging (Fig. 1d and f, respectively). Liver showed prominent uptake of the probe on PET imaging at 24 h (Fig. 1d).

Ex vivo nuclear and optical imaging. Ex vivo study was performed on harvested tissues and the biodistribution experiment showed the uptake of ${ }^{64} \mathrm{Cu}$-DOTA-NuB2-Alexa Fluor 750 in tumor and other organs as determined by radioactivity and fluorescence intensity (Fig. 2A and B,
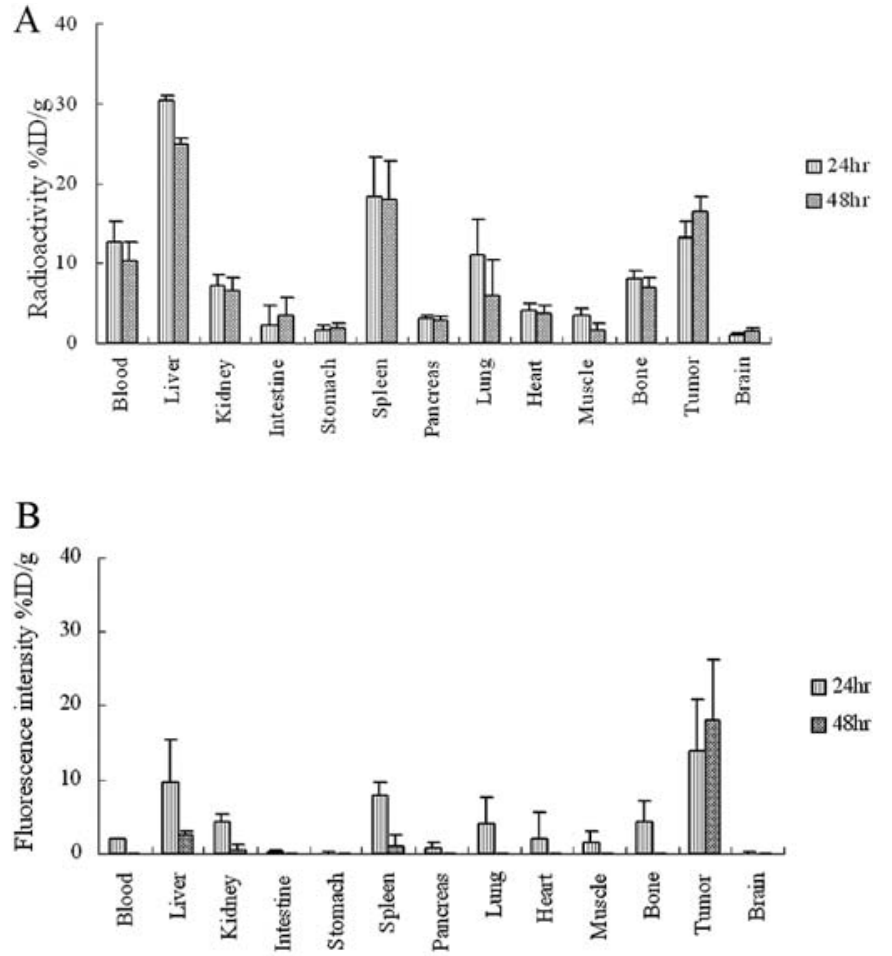

Figure 2. Ex vivo biodistribution of ${ }^{64} \mathrm{Cu}$-DOTA-NuB2-Alexa Fluor 750 in Raji lymphoma xenografts (A) measured by radioactivity and (B) measured by fluorescence intensity at 24 and $48 \mathrm{~h}$ after injection. Groups of 4 mice were sacrificed at each time point. Mean and standard deviations have been corrected for physical decay of ${ }^{64} \mathrm{Cu}$.

respectively). The radioactivity in the liver activity reached a plateau at $24 \mathrm{~h}(30.28 \pm 0.76)$ point owing to non-specific uptake of the probe by the reticuloendothelial system (RES) but dropped gradually over time $(48 \mathrm{~h}, 24.88 \pm 1.10)$. The activity of liver measured by the fluorescence was high at $24 \mathrm{~h}(9.62 \pm 5.67)$, and dropped steadily at $48 \mathrm{~h}(2.50 \pm 0.51)$. The absolute signal intensity from NIRF is quite different than that of PET signal. This is likely due to the decomposition of DOTA complex and trapping of ${ }^{64} \mathrm{Cu}$ inside the tumor or degradation of the fluorophore thus decreasing the fluorescence intensity. The uptake measured by the radioactivity vs. fluorescence intensity in other organs such as muscle was very low at $24 \mathrm{~h}(3.41 \pm 1.20$ vs. $1.41 \pm 1.60)$ and $48 \mathrm{~h}$ $(1.63 \pm 0.33$ vs. 0.00$)$. The uptake of the probe in tumor increased with time, it was $3.19 \pm 0.58$ vs. $0.25 \pm 0.30$ at $30 \mathrm{~min}$, $13.09 \pm 2.01$ vs. $13.73 \pm 7.00$ at $24 \mathrm{~h}$ and reached a peak at $48 \mathrm{~h}$ post-injection $(16.34 \pm 2.75$ vs. $17.92 \pm 8.12)$. Significantly higher radioactivity or fluorescence intensity was observed in tumor than that of muscle at any time point $(\mathrm{p}<0.001)$. When the mice were injected with DOTA-Alexa Fluor 750, the uptake in the tumor was significantly lower $(\mathrm{p}<0.05$, data not shown) than that of DOTA-NuB2-Alexa Fluor 750 as shown in the Fig. 3.

Strong correlation was obtained between the organs-tomuscle ratios of liver, kidney, intestine, spleen, pancreas, lung, heart, bone and tumor measured by two different modalities $(\mathrm{p}<2.2 \mathrm{e}-16, \mathrm{r}=0.94)$ as shown in the Fig. 4. A significant correlation was obtained between the radioactivity and fluorescence intensity in all organs at each time point evaluated (data not shown). 


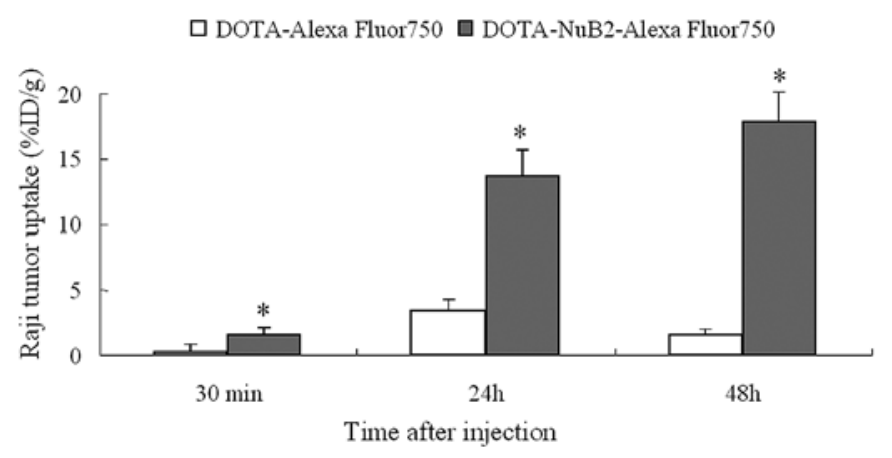

Figure 3. Raji tumor uptake of DOTA-Alexa Fluor 750 and ${ }^{64} \mathrm{Cu}-\mathrm{DOTA}-$ NuB2-Alexa Fluor 750 over time, as quantified by ex vivo analysis measured by fluorescence intensity ( $n=3$ per time point). Significant difference was noted at each time point; ${ }^{*} \mathrm{P}<0.05$.

\section{Discussion}

The development of mAb-based imaging agent that can produce detectable signal in multimodal imaging system such as PET and optical imaging would facilitate the clinical application of optical molecular probes, while limiting the toxicity of the probe. The present study demonstrated that ${ }^{64} \mathrm{Cu}$-DOTA-NuB2-Alexa Fluor 750 exhibits strong and specific binding to the tumor expressing CD20 antigen as confirmed by the ex vivo biodistribution study and in vivo PET and NIRF imaging. The tumor uptake was typically high with lower RES uptake and showed better pharmacokinetics when compared with the previous dual-labeled studies with peptides. Development of a mAb-based dual functional imaging agent containing both NIRF and radionuclides for PET could facilitate the translation of NIRF imaging into clinical applications to provide synergistic advantages over single imaging modality (13). A strong correlation between NIRF and PET imaging can be assured, as both NIRF and PET are tomographic modalities (14). Thus, the development of a dual-labeled probe offers a convenient and direct approach for accurately assessing the pharmacologic properties of NIRF in preclinical studies (15).

Previously quantum dots (QD) based dual function probe for imaging of tumor with PET and NIRF has been reported $(8,9)$. However, the present large-sized QDs are ineffective for in vivo targeting of the tumor receptor and have rapid uptake in the RES and slow renal clearance (14). The synthesis of small-sized QDs that are highly stable and biologically effective is critical. The toxicity of cadmium present in QD and the huge molecular structure of the QD resulting in the high RES uptake and low target uptake cannot be ignored when considering application in the clinical practice.

The present study demonstrated significantly higher uptake in the tumor than that in the muscle region at 24 and $48 \mathrm{~h}$ post-injection with ${ }^{64} \mathrm{Cu}$-DOTA-NuB2-Alexa Fluor 750 . When mice were imaged with DOTA-Alexa Fluor 750, the uptake in tumor was significantly lower at $30 \mathrm{~min}, 24 \mathrm{~h}$ and $48 \mathrm{~h}$ compared with DOTA-NuB2-Alexa Fluor 750, which excluded the possibility of non-specific binding of the antigen to the free DOTA-Alexa Fluor 750. The liver signal intensity as

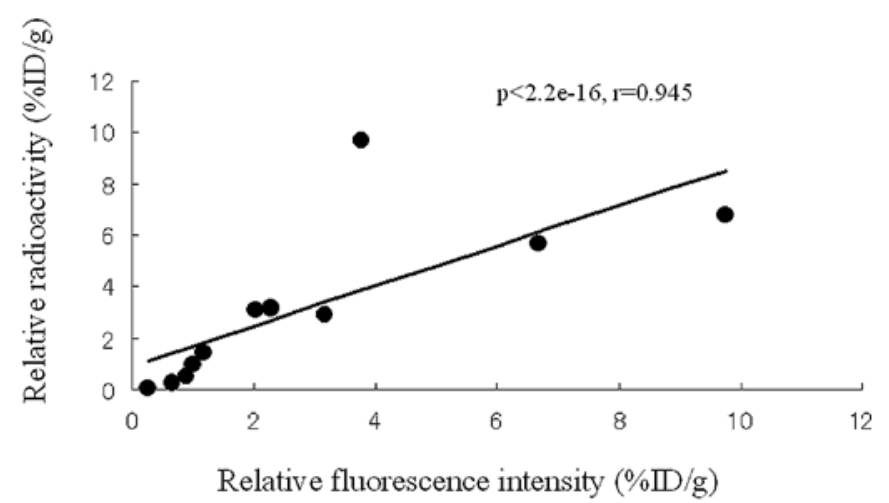

Figure 4. Correlation between radioactivity and fluorescence intensity of ${ }^{64} \mathrm{Cu}$-DOTA-NuB2-Alexa Fluor 750 at $24 \mathrm{~h}$ after injection. Ex vivo organs to muscle ratios of liver, kidney, intestine, stomach, spleen, pancreas, lung, heart, bone, and tumor are demonstrated.

demonstrated by PET and NIRF was higher than that of tumor or muscle. The presence of Fc receptor in the liver might have lead to the non-specific binding of intact $\mathrm{mAb}$ in which the Fc portion is not removed (15). However, the signal intensity decreased after forming a plateau at $24 \mathrm{~h}$, which allowed sufficient time for the probe to reach the target. It confirmed that the probe could extravasate to reach the receptor targets expressed on the surface of the tumor cells, thus, enhancing the specific targeting and enabling the escape from the RES (14). The extravasation study should be verified in our future studies with intravital microscopy. The clearance of ${ }^{64} \mathrm{Cu}$-DOTA-NuB2-Alexa Fluor 750 is usually through the hepatic pathway like any other mAb-based imaging agent. Even though the in vivo studies showed better results the ${ }^{64} \mathrm{Cu}$-DOTA-NuB2-Alexa Fluor 750 is a stable compound, ${ }^{64} \mathrm{Cu}$-DOTA-mAb is reported to dissociate from the chelator and the free ${ }^{64} \mathrm{Cu}$ accumulates in the liver where it is incorporated into endogenous copper binding proteins such as ceruloplasmin and superoxide dismutase $(14,16)$ which shows higher uptake in the liver by PET and might cause the discrepancy between PET and NIRF. Alternatively, one could use more stable chelating agents with ${ }^{64} \mathrm{Cu}$ to obtain better pharmacokinetics. This approach is currently under investigation in our laboratories. The present study is based on the assumption that the organ activity of PET shows true measurement of well counting and this has been validated in previous studies (17) and the distribution of ${ }^{64} \mathrm{Cu}$ is a true reflection of the localization of NIRF imaging agent in various organs as supported by previous studies $(8,9,14)$.

In conclusion, we successfully developed a mAb-based dual functional probe, ${ }^{64} \mathrm{Cu}-\mathrm{DOTA}-\mathrm{NuB} 2-$ Alexa Fluor 750 which can be characterized for in vivo imaging of the CD20 expression for early and sensitive lesion detection, patient selection for better treatment monitoring and dose optimization. A statistically significant correlation was observed between radioactivity and fluorescence intensity, confirming the stability of the dual functional probe, thus raising the probability facilitating the integration of optical imaging into existing PET system thereby accelerating the use of the combined strength of both modalities. 


\section{References}

1. Gurfinkel M, Ke S, Wen X, Li C and Sevick-Muraca EM: Nearinfrared fluorescence optical imaging and tomography. Dis Markers 19: 107-121, 2003-2004.

2. Mahmood U, Tung CH, Bogdanov A Jr and Weissleder R: Near-infrared optical imaging of protease activity for tumor detection. Radiology 213: 866-870, 1999.

3. Ke S, Wen X, Gurfinkel M, Charnsangavej C, Wallace S, Sevick-Muraca EM and Li C: Near-infrared optical imaging of epidermal growth factor receptor in breast cancer xenografts. Cancer Res 63: 7870-7875, 2003.

4. Anderson CJ, Dehdashti F, Cutler PD, Schwarz SW, Laforest R, Bass LA, Lewis JS and McCarthy DW: ${ }^{64} \mathrm{Cu}$-TETA-octreotide as a PET imaging agent for patients with neuroendocrine tumors. J Nucl Med 42: 213-221, 2001.

5. Culver J, Akers W and Achilefu S: Multimodality molecular imaging with combined optical and SPECT/PET modalities. J Nucl Med 49: 169-172, 2008.

6. Li C, Wang W, Wu Q, Ke S, Houston J, Sevick-Muraca E, Dong L, Chow D, Charnsangavej C and Gelovani JG: Dual optical and nuclear imaging in human melanoma xenografts using a single targeted imaging probe. Nucl Med Biol 33: 349-358, 2006

7. Wang W, Ke S, Kwon S, Yallampalli S, Cameron AG, Adams KE, Mawad ME and Sevick-Muraca EM: A new optical and nuclear dual-labeled imaging agent targeting interleukin 11 receptor alpha-chain. Bioconjug Chem 18: 397-402, 2007.

8. Cai W, Chen K, Li ZB, Gambhir SS and Chen X: Dual-function probe for PET and near-infrared fluorescence imaging of tumor vasculature. J Nucl Med 48: 1862-1870, 2007.

9. Chen K, Li ZB, Wang H, Cai W and Chen X: Dual-modality optical and positron emission tomography imaging of vascular endothelial growth factor receptor on tumor vasculature using quantum dots. Eur J Nucl Med Mol Imaging 35: 2235-2244, 2008.
10. Sampath L, Kwon S, Ke S, Wang W, Schiff R, Mawad ME and Sevick-Muraca EM: Dual-labeled trastuzumab-based imaging agent for the detection of human epidermal growth factor receptor 2 overexpression in breast cancer. J Nucl Med 48: 1501-1510, 2007.

11. Lewis MR, Wang M, Axworthy DB, Theodore LJ, Mallet RW, Fritzberg AR, Welch MJ and Anderson CJ: In vivo evaluation of pretargeted ${ }^{64} \mathrm{Cu}$ for tumor imaging and therapy. J Nucl Med 44: 1284-1292, 2003.

12. Oriuchi N, Higuchi T, Hanaoka H, Iida Y and Endo K: Current status of cancer therapy with radiolabeled monoclonal antibody. Ann Nucl Med 19: 355-365, 2005.

13. Cai W and Chen X: Multimodality molecular imaging of tumor angiogenesis. J Nucl Med 49: S113-S128, 2008.

14. Schipper ML, Cheng Z, Lee SW, Bentolila LA, Iyer G, Rao J, Chen X, Wu AM, Weiss S and Gambhir SS: microPET-based biodistribution of quantum dots in living mice. J Nucl Med 48: 1511-1518, 2007.

15. Anderson CJ, Connett JM, Schwarz SW, Rocque PA, Guo LW, Philpott GW, Zinn KR, Meares CF and Welch MJ: Copper-64labeled antibodies for PET imaging. J Nucl Med 33: 1685-1691, 1992.

16. Bass LA, Wang M, Welch MJ and Anderson CJ: In vivo transchelation of copper-64 from TETA-octreotide to superoxide dismutase in rat liver. Bioconjug Chem 11: 527-532, 2000.

17. Knoess C, Siegel S, Smith A, Newport D, Richerzhagen N, Winkeler A, Jacobs A, Goble RN, Graf R, Wienhard K and Heiss WD: Performance evaluation of the microPET R4 PET scanner for rodents. Eur J Nucl Med Mol Imaging 30: 737-747, 2003. 